\title{
Building Galactic Disks in Triaxial Dark Matter Halos
}

\author{
Isaac Shlosman \\ Department of Physics \& Astronomy, University of Kentucky, Lexington, KY 40506, USA \\ email: shlosman@pa.uky.edu
}

\begin{abstract}
We review our recent work on the formation and evolution of disks within triaxial dark matter (DM) halos by means of numerical simulations, including star formation and feedback from stellar evolution. The growing disks are strongly influenced by shapes of DM halos and modify them in turn. Disk parameters are in a broad agreement with those in the local universe. Gas-rich stellar bars grow in tandem with the disk and facilitate the angular momentum redistribution in the system and radial gas inflow. Nested bars appear to form as a by-product. Interactions between various non-axisymmetric components-bars, disks and halos lead to decay of bars or washing out of ellipticity in the inner halo.
\end{abstract}

Keywords. galaxies: evolution - galaxies: formation - galaxies: halos - galaxies: kinematics and dynamics - galaxies: starburst - galaxies: structure

\section{Introduction}

Fundamental difficulties remain in our understanding of structure formation on the subgalactic scales. Among these, formation and evolution of galactic disks and bulges, angular momentum redistribution between baryonic and dark matter (DM), radial density profiles of DM halos (especially survival of the DM cusps), fueling of the central activity, correlation between masses of the central black holes (SBHs) and the surrounding host galaxies - provide an incomplete but impressive list of stand-by problems. On the other hand, understanding the origin of the large-scale structure in the universe has been largely successful.

Because the formation and evolution of galactic disks involves grossly non-linear processes, both dissipative and non-dissipative, they are typically investigated by means of extensive numerical simulations. A major outcome of these simulations is that halos forming within the $\Lambda \mathrm{CDM}$ cosmology appear to be universally triaxial (e.g., Allgood et al. 2006) while those inferred in the nearby universe are compatible with being axisymmetric (e.g., Combes 2002). Here we argue that the underlying halo shape evolution is directly related to the disk formation and its subsequent growth.

Two main approaches have been used to follow the formation and evolution of disk galaxies. First, large-scale cosmological simulations of collisionless and collisional matter have been invoked, with a subsequent extraction of a DM halo and its immediate environment. Resampling was implemented in order to increase the number of particles per halo (e.g., Sommer-Larsen et al. 2003; Governato et al. 2006). With all the advantages, these simulations still suffer from insufficient numerical resolution and cannot compete with simulations of individual galaxies. Second, models of galactic disks in frozen sphericallysymmetric halos or analytically growing (frozen) halos have been advanced (e.g., Samland \& Gerhard 2003; Immeli et al. 2004). They treat a variety of important processes, such as chemical evolution, but cannot follow other processes, such as angular momentum redistribution in the system, especially the halo-disk resonant coupling. 


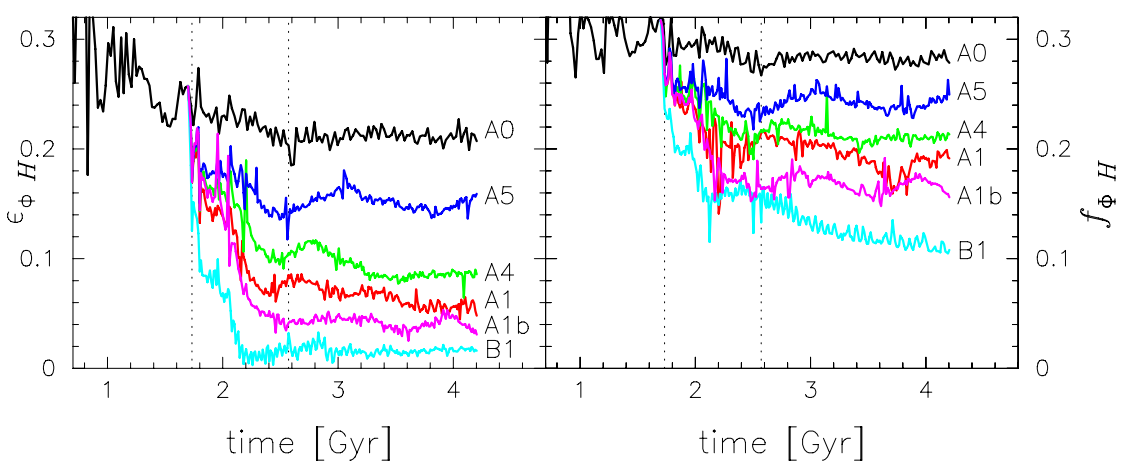

Figure 1. Evolution of the halo equatorial potential ellipticity (left) and potential flatness(right) at representative $5 \mathrm{kpc}$ in different model realizations: A0 (pure DM), A1 (growing disk), A4 (slowly growing disk), A5 (nongrowing seed disk), A1b (frozen disk), B1 (massive growing disk). Vertical dotted lines (from left to right): $z=3$ and 2 .

Here we summarize our results from two alternative approaches: (1) simulations of a limited volume using cosmological initial conditions obtained by means of Constrained Realizations method (Hoffman \& Ribak 1991) of a prescribed density field (Romano-Diaz et al. 2006, 2007). Berentzen \& Shlosman (2006) have applied this method in order to follow the evolution of DM within a $4 h^{-1} \mathrm{Mpc}^{3}$ box, starting with a redshift $z=120$ and inserting a 'seed' disk at $z=3$, in the open CDM (OCDM) Universe with $\Omega_{0}=0.3, h=$ 0.7 and $\sigma_{8}=0.9$. The disk was grown tenfold over different periods of time, $1-3$ Gyr. (2) Heller, Shlosman \& Athanassoula (in preparation) have followed the Hubble expansion and collapse of an isolated DM+baryons perturbation starting with $z=36$. Our star formation (SF) algorithm is physically motivated and feedback from stellar evolution in the form of OB stellar winds and SN is included. We have used the updated hybrid $\mathrm{SPH} / N$-body code FTM-4.4 of Heller \& Shlosman.

\section{Galaxy Models from Constrained Realizations}

The DM model has been constructed with a nested set of three off-center $2.5-3.5 \sigma$ perturbations. The pure DM halo (model A0) went through the epoch of major mergers which ended at $z \sim 7$. Its density profile is given by NFW (Navarro et al. 1997) established by $z \sim 10$. We use the isopotential contours to measure the halo shape, which has a substantial advantage over the isodensities method (Berentzen et al. 2006). The A0 model has settled in a stable, substantially triaxial (elliptical and flat) configuration (Fig. 1). This halo ellipticity leads to a strong interaction with a seed disk immersed at $z=3$ and grown (or not) over the next 1-3 Gyr.

We find that a growing disk is responsible for washing out the halo equatorial ellipticity (in the disk plane) and for diluting its flatness over a period of time comparable to the disk growth. In models where the disk contributes more to the overall rotation curve, it appears more efficient in axisymmetrizing the halo, without accelerating the halo figure rotation. The observational corollary is that the maximal disks probably reside in nearly axisymmetric halos, while disks whose rotation is dominated by the halo at all radii can reside in more elliptical halos. The halo shape is sensitive to the final disk mass, but is independent of how the seed disk is introduced into the system-abruptly or quasi-adiabatically. It is only weakly sensitive to the timescale of the disk growth. While models with massive disks have developed strong bars, we find that light disks have this instability damped by the halo triaxiality - an interesting implication for the cosmological 

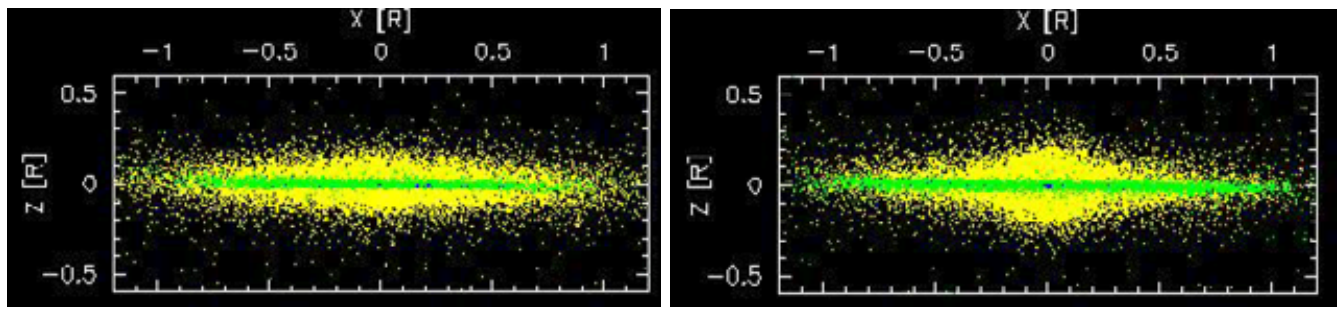

Figure 2. Examples of nearly bulgeless (left) and bulge-dominated (right) edge-on disks. Colors: green (gas), yellow (stars), blue (star formation), DM (omitted). Units: [1] $=10 \mathrm{kpc}$.

evolution of disk galaxies. The halo itself responds to the stellar bar by developing a gravitational wake - a 'ghost' bar of its own which is almost in-phase with that in the disk.

\section{Galaxy Models from Isolated Perturbations}

We have also investigated formation and evolution of galactic disks within assembling live DM halos in a large number of models (Heller et al., in preparation). Disk and halo structural components have been evolved from the cosmological initial conditions and represent the collapse of an isolated density perturbation in the OCDM Universe with $\Omega_{0}=0.3, h=0.7$ and $10 \%$ baryons. The gas temperature was obtained from the energy equation. The SF takes place in the Jeans unstable, contracting region. We fix the gasto-background density at which the gas is converted to a star, the local collapse-to-free fall time, and introduce the probability that the gas particle produces a star during a given timestep. Four generations of stars form per gas particle with an instantaneous recycling along with an increment in metallicity. The balance of the specific internal energy along with the gas ionization fractions of $\mathrm{H}$ and $\mathrm{He}$ and the mean molecular weight are computed as a function of $\rho$ and $T$ for an optically thin primordial composition gas.

The energy feedback from the stellar evolution includes the OB stellar winds and SN, and uses the thermalization parameter - a fraction of the feedback deposited as a thermal energy and converted into kinetic energy via equations of motion. Full details of the SF algorithm and energy feedback are given elsewhere. The initial density profile corresponds to the average density around a $2 \sigma$ peak in a Gaussian random density field. The spin parameter is set to $\lambda=0.05$. We impose the collapse redshift of $z=2$. The mass of collisionless DM particles is $7 \times 10^{11} \mathrm{M}_{\odot}$ and the gas comprises $10 \%$ of the total mass initially.

We find that the pure DM models lead to the formation of triaxial halos with a negligible tumbling of $\sim \pi$ over the Hubble time. This leads to corotation and inner Lindblad resonance being moved to very large and small radii, respectively. Orbital response of a disk immersed in such a halo will be always out of phase with the halo major axis - thus diluting the halo equatorial potential and washing out its ellipticity. (Baryon effect on the halo shape was first noticed by Dubinski [1994] by diluting the halo potential with that of a rigid Plummer sphere, and by Kazandzidis et al. [2004] by damping baryons inside the halo.) In addition, the increasing central mass concentration in the model contributes to the DM orbit scattering and so to decrease in halo ellipticity and flattening.

Evolution of the DM halos differs in the presence of baryons in that it reaches smaller triaxialities and these are reduced further. Consequently, the growing disks exhibit substantial ellipticities in the first $2 \mathrm{Gyr}$. These heavily gas rich disks respond dramatically to halo's non-axisymmetric driving by forming a pair of strong grand-design density waves 
in the stellar component and associated shocks in the gas. The pattern speeds of the oval disks and the barlike response are identical and lead to a rapid gas inflow toward the central kpc. In a number of models, this resulted in decoupling the central part of the bar in the prograde direction - i.e., formation of nested bars (Heller, Shlosman \& Athanassoula $2006)$ in accord with Shlosman et al. $(1989,1990)$ but under different initial conditions (Begelman et al. 2006).

The disk grows from inside out by increasing its radial scalelength. This and other disk parameters appear to lie within the range observed in the local universe-from essentially bulgeless to bulge dominated disks (Fig. 2). They do not 'suffer' from the angular momentum 'catastrophe.' The total $J$ is approximately conserved, even in models accounting for feedback from the stellar evolution. The baryons lose $\sim 30 \%$ and the DM acquires $\sim 3 \%$ of their original $J$. The specific $J$ for the DM is nearly constant while that for the baryons is decreasing with time.

In summary, shapes of host DM halos are shown to have a profound effect on the morphology and dynamics of growing disks - with the caveat that the disk growth alters the halo shape. We find that maximal disks wash out the equatorial ellipticity of inner $(<20 \mathrm{kpc})$ halos and lessen their flatness. They are expected to reside in (nearly) axisymmetric halos in the local universe, while disks dominated by halos at all radii can reside in elliptical halos. The initial disk response to the halo asymmetry is barlike. This facilitates the angular momentum transfer between the disk and the host halo, and triggers the radial gas inflow. These conditions provide fertile grounds for the formation of gas rich nested bars. Whether the large-scale bars survive for longer time periods depends largely on the timescale of a halo losing its ellipticity, as stellar bars and elliptical halos appear to be incompatible (El-Zant \& Shlosman 2002).

\section{Acknowledgements}

I am indebted to my collaborators Lia Athanassoula, Ingo Berentzen, Clayton Heller, Yehuda Hoffman, Shardha Jogee and Emilio Romano-Diaz on these issues. The relevant grants from NASA and NSF are gratefully acknowledged.

\section{References}

Allgood, B., et al. 2006, MNRAS, 367, 1781

Begelman, M.C., Volonteri, M. \& Rees, M.J. 2006, MNRAS, 370, 289

Berentzen, I., Shlosman, I. \& Jogee, S. 2006, ApJ, 637, 582

Berentzen, I. \& Shlosman, I. 2006, ApJ, 648, 807

Combes, F. 2002, New Astron. Rev., 46, 755

Dubinski, J. 1994, ApJ, 431, 617

El-Zant, A. \& Shlosman, I. 2002, ApJ, 577, 626

Governato, F., et al. 2006, MNRAS, submitted (astro-ph/0602351)

Heller, C.H., Shlosman, I. \& Athanassoula, E. 2006, ApJ Lett., subm. (astro-ph/0610428)

Hoffman, Y. \& Ribak, E. 1991, ApJ, 380, L5

Immeli, A., et al. 2004, A\&A, 413, 547

Kazandzidis, S., et al. 2004, ApJ, 611, L73

Navarro, J.F., Frenk, C.S. \& White, S.D.M. 1997, ApJ, 490, 493

Romano-Diaz, E., et al. 2006, ApJ, 637, L93

Romano-Diaz, E., et al. 2007, ApJ, 655, in press (astroph/0610090)

Samland, M. \& Gerhard, O. 2003, A\&A, 399, 961

Shlosman, I., Frank, J. \& Begelman, M.C. 1989, Nature 338, 45

Shlosman, I., Begelman, M.C. \& Frank, J. 1990, Nature 345, 679

Sommer-Larsen, J., Gotz, M. \& Portinari, L. 2003, ApJ, 596, 47 


\section{Discussion}

BRIAN LEWIS: Would a super-massive black hole in the galaxy change any of your conclusions?

IsaAc Shlosman: The outer disk and the large-scale stellar bar evolution do not depend on the central BH. Disk galaxies host BHs that are 10 times less massive than required to dissolve the bars. Of course, if we believe the observational determination of $\mathrm{BH}$ masses. The BH can have an important role in determining the physical processes in the central kpc, i.e. bulge properties. There can be a close relation between the BH mass, the surrounding bulge, and the host dark matter halo (e.g. El Zant et al. 2003).

AndREAS Just: What is the argument to get a maximum disk, in the disk-halo interaction?

IsAaC Shlosman: More massive disks have larger effect on the inner halo diluting its equatorial prolateness. Maximal disks are dominating the inner rotation curve. In all such cases, they have a profound effect on the inner (and even outer) halo shapes. The inner halo becomes oblate.

Ben MoOre: Do you understand why dissipation causes the halo to change shape?

IsaAC Shlosman: Dissipation has no effect on the halo shape. In a (nearly) non-rotating halo potential, gas or stellar fluid response is the same-perpendicular to the halo's major axis. Such a response generates a mass distribution which dilutes the dark matter equatorial prolateness. Additional factor is that the disk sits at the bottom of a DM potential well, thus scattering orbits of DM particles. Again, this has nothing to do with dissipation. 